\title{
In memoriam Rudolf Meyer
}

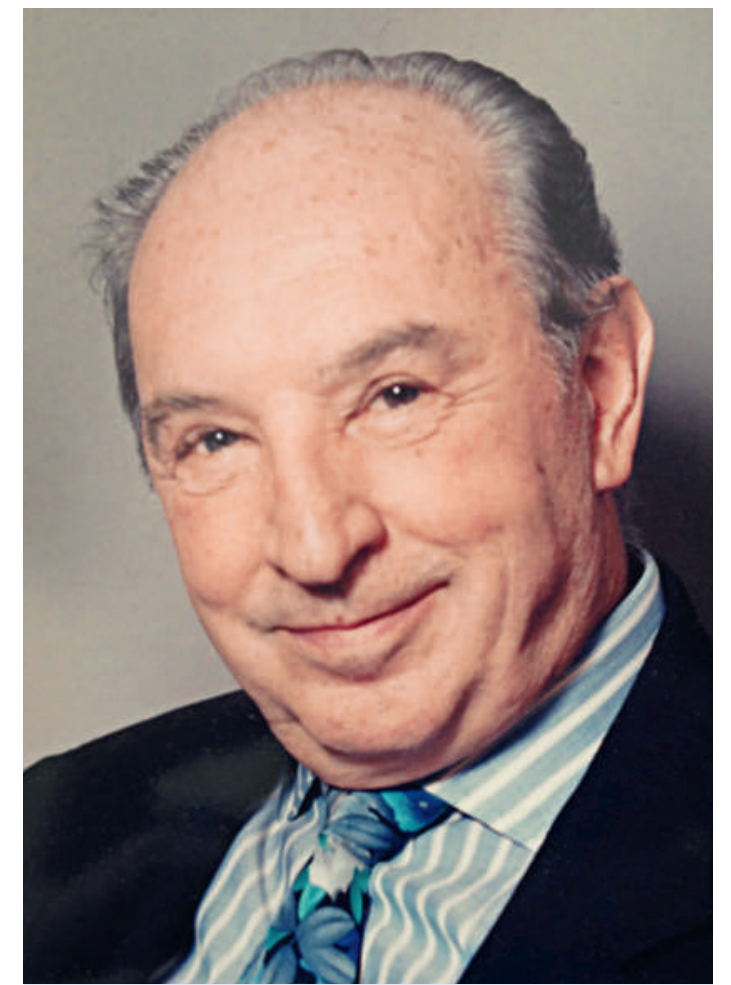

Prof. Dr Rudolf Meyer

$(\dagger 2013)$

Figure marquante dans son domaine, la chirurgie plastique et reconstructive, le Prof. Dr Rudolf Meyer, 94 ans, a fermé ses yeux au Centre Hospitalier Universitaire Vaudois CHUV, ou il avait été hospitalisé. Le Prof. Meyer, qui a étudié la médecine à Bâle, marié et père de deux fils et d'une fille, avait acquis une stature internationale.
Arrivé à Lausanne en 1962 comme médecin ORL à l'Hôpital cantonal, il fut le premier à enseigner la chirurgie plastique dans une Université, celle de Lausanne. Par la suite, il fut cofondateur de la Société suisse de chirurgie plastique et de la société internationale de la discipline, l'ISAPS.

«A l'époque, cette spécialité n'existait pas», témoigne le Dr Jean-François Emeri, qui a pratiqué aux côtés du Prof. Meyer au sein du Centre de chirurgie plastique de Lausanne, ouvert par le défunt (avec le Dr Kesselring) en 1977.

Spécialiste de rhinoplastie, le Prof. Meyer a accueilli dans son cabinet des patients venus du monde entier. «ll a inventé la chirurgie du nez, l'une des plus complexes qui soit», affirme le Dr Emeri qui se souvient de cette «personnalité attachante». Lors d'interviews, on percevait, derrière son sourire exprimant le goût de la vie et le plaisir au travail, une véritable passion pour son métier. «C'était sa raison de vivre», confirme le Dr Emeri. Le Prof. Meyer, qui a manié le scalpel jusqu'à un âge avancé, a retouché de nombreux nez opérés une première fois sans grand succès. Il pratiquait aussi les délicates opérations du changement de sexe chez des transsexuels hommes désireux de devenir des femmes. Appelé dans le monde entier, il a aussi beaucoup opéré à l'étranger.

Grand sportif, le Prof. Dr Meyer s'est aussi adonné à la peinture dès sa jeunesse, un art qu'il a pratiqué ces dernières années et qu'il a présenté lors de diverses expositions.

En 2012, il a fait don de sa bibliothèque de travail au CHUV, qui a le projet de nommer une salle en hommage à celui qui a jeté les bases de la chirurgie plastique dans l'hôpital vaudois.

Francine Brunschwig

(Source: 24 heures, 8 mai 2013) 